\title{
Stable Model for Active Contour based Region Tracking using Level Set PDE
}

\author{
Suk-Ho Lee, Member, KIICE
}

\begin{abstract}
In this paper, we propose a stable active contour based tracking method which utilizes the bimodal segmentation technique to obtain a background color diminished image frame. The proposed method overcomes the drawback of the Mansouri model which is liable to fall into a local minimum state when colors appear in the background that are similar to the target colors. The Mansouri model has been a foundation for active contour based tracking methods, since it is derived from a probability based interpretation. By stabilizing the model with the proposed speed function, the proposed model opens the way to extend probability based active contour tracking for practical applications.
\end{abstract}

Index Terms-Tracking, Active Contour, Segmentation, Level Set.

\section{INTRODUCTION}

IN this paper, we propose a stable tracking model which is an extension of the Mansouri model [1] that has been proposed for region tracking without motion computation. Tracking regions in an image sequence is a challenging and difficult problem in image processing and computer vision. However, region tracking has also many important applications such as intelligent video surveillance, automated video editing, video database search and retrieval, etc.

Recently, active contour based approaches have been studied for the aim of simultaneous tracking and segmentation of the object [1]-[7]. The approach aims for obtaining the object boundary for further high level computer vision tasks, while tracking the object. If the boundary of the object is known, then intelligent analysis can be done of the situation in which the object is involved (See Figure 1).

The Mansouri model [8] has been proposed for region tracking that derives from a Bayesian formulation which uses no motion field or motion parameters computation. This model has become a foundation for many active contour based tracking models since the problem of region tracking is formulated as a Bayesian estimation problem, and the resulting tracking algorithm is expressed as a level set partial differential equation.

\footnotetext{
Manuscript received October 10, 2011; revised November 17, 2011; accepted November 30, 2011.

Suk-Ho Lee is with the Department of Software Engineering, Dongseo University, Busan, 617-716, Korea (Email: petra@gdsu.dongseo.ac.kr)
}

Until this paper, other active contour based algorithms just used motion information (optical flow), or background subtraction to define the speed function of the active contour.

Another novelty of the Mansouri model is that very little a priori information about the region being tracked is used in the algorithm. This kind of expression has opened a wide possibility for interpreting the tracking process as partial differential equation solving problem. However, the Mansouri model is rather sensitive to the color variation in the background. If a certain color appear in the background such which is similar to the color of the object, then the active contour tries to include the background region also in the target region, and result in a failure of the tracking.

In this paper, we propose a new speed function which is an extension of the speed function of that used in the Mansouri model to stabilize the tracking process. We also use a bimodal segmentation technique to obtain a new image in which the target region is better discriminated from the background. We unify both techniques in a single framework. Experimental results verifying the stability of the proposed model are given at the end of this paper.

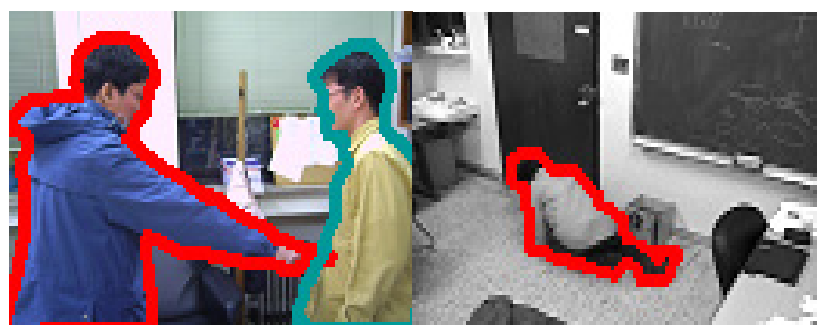

Fig. 1. Examples when the boundary of the object can give some clue to the situation of the scene.

\section{MATHEMATICAL FORMULATION FOR PROBABILITY BASED ACTIVE CONTOUR MOTION TRACKING : THE MANSOURI MODEL}

In this section, we introduce how the Mansouri model results from a probability based formulation of the active contour based motion tracking. We follow the main process described in [7]. Then, we refer to the problems with the Mansouri model. In the probabilistic approach, 
the shape and the motion parameters of the active contour are updated such that the contour's a posteriori probability is maximized. To formulate the posteriori probability function, it is assumed that the image is composed of the object region and the background region.

$$
R=R_{o b j} \cup R_{b c k},
$$

where $R$ corresponds to the full image region and $R_{o b j}$ and $R_{b c k}$ correspond to the object and the background regions, respectively. The likelihood of observing the boundary $\Gamma$ is equal to the likelihood of partitioning the image into the object and the background regions:

$$
P(\Gamma)=P(\varphi(R))=\left\{R_{o b j}, R_{b c k}\right\},
$$

where $\varphi$ is the partitioning operator. The object tracking problem is formulated in terms of the boundary probability, $P_{\Gamma}$, defined by the probabilities of the regions, given the current image, $I^{n}$, and the previous collection of object boundaries, $\Gamma^{i}: i=0,1, \ldots . n-1$, as

$$
P_{\Gamma}=P\left(\varphi\left(R^{n}\right) \mid I^{n}, \Gamma^{n-1}, \Gamma^{n-2}, \ldots . \Gamma^{0}\right) .
$$

Then using the Bayes' rule, this can be reformulated as

$$
P_{\Gamma}=\frac{P\left(I^{n} \mid \varphi\left(R^{n}\right), \Gamma^{n-1}, \Gamma^{n-2}, \ldots \Gamma^{0}\right) P\left(\varphi\left(R^{n}\right) \mid \Gamma^{n-1}, \Gamma^{n-2}, \ldots \Gamma^{0}\right)}{P\left(I^{n} \mid \Gamma^{n-1}, \Gamma^{n-2}, \ldots \Gamma^{0}\right)}(3)
$$

Here, the first term in the numerator can be split into two terms such as

$$
P\left(I^{n} \mid R_{o b j}^{n}, \Gamma^{n-1}, \Gamma^{n-2}, \ldots . \Gamma^{0}\right)
$$

and

$$
P\left(I^{n} \mid R_{b c k}^{n}, \Gamma^{n-1}, \Gamma^{n-2}, \ldots . \Gamma^{0}\right)
$$

Using the assumption that each pixel in the image is independent of each other such that:

$$
\begin{aligned}
& P_{\Gamma}=\frac{\prod_{R_{o b j}} P_{o b j}\left(I^{n}\right) \prod_{R_{b c k}} P_{b c k}\left(I^{n}\right)}{C} \\
& =\frac{\prod_{x_{1}}\left[\prod_{x_{2}} P_{o b j}\left(I^{n}\left(x_{2}\right)\right) \prod_{x_{3}} P_{b c k}\left(I^{n}\left(x_{3}\right)\right)\right]}{C}
\end{aligned}
$$

Here, $x_{1}, x_{2}, x_{3}$ are pixels that lie in a small patch which center is on the active contour, each corresponding to the inside, the boundary and the outside of the active contour (Fig. 2). Then by maximizing this posteriori probability, we obtain the Energy functional for tracking:

$$
E(\Gamma)=\iiint_{x_{1}}\left[\iint_{x_{2}} \Psi_{o b j}\left(x_{2}\right) d x_{2}+\iint_{x_{3}} \Psi_{b c k}\left(x_{3}\right) d x_{3}\right] d x_{1},
$$

Where

$\Psi_{o b j}(x)=-\log P_{R_{o b j}}\left(I^{n}(x)\right), \quad \Psi_{b c k}(x)=-\log P_{R_{b c k}}\left(I^{n}(x)\right)$

The speed function of the Mansouri model is computed by minimizing the energy functional in (7). By reformulating (7), the speed function $F$ is computed based on the relationship between the intensity values of the background pixels and the foreground pixels inside a local window.

$$
F=\iint_{x \in \Omega}\left(\Psi_{o b j}(x) \chi_{o b j}-\Psi_{b c k}(x) \chi_{b c k}\right) d x,
$$

where

$\Psi_{o b j}(x)=-\log P_{R_{o b j}}\left(I^{n}(x)\right), \quad \Psi_{b c k}(x)=-\log P_{R_{b c k}}\left(I^{n}(x)\right)$

with

$$
P_{\alpha}(x)=\max _{z:\|z\| \leq m} e^{-\frac{\left(I^{n-1}(x)-I^{n}(x+z)\right)^{2}}{2 \sigma^{2}}} .
$$

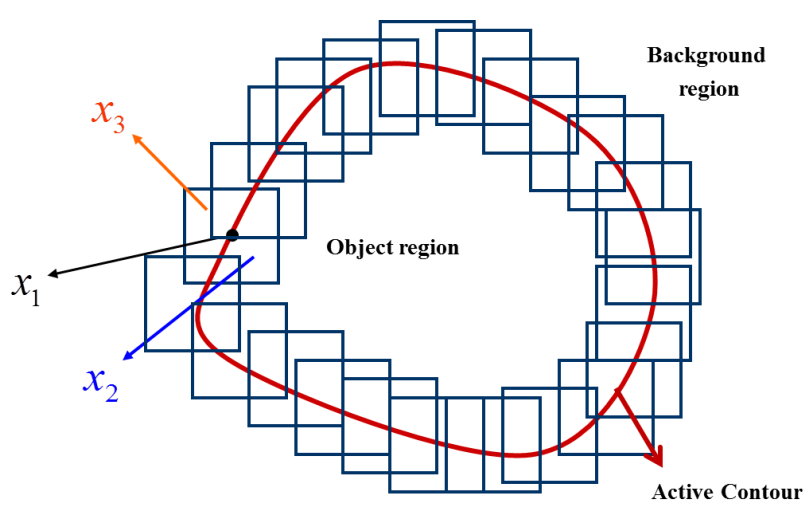

Fig. 2. Pixels inside the local patch along the active contour.

The speed function decides the speed of the following curve evolution equation:

$$
\frac{d C}{d t}=F \vec{N}
$$

which evolves the curve $C$ according to the speed field $F$ in the normal direction $\vec{N}$. In the classical level-set method this evolution is achieved by numerically solving the following PDE

$$
\phi_{t}=-F_{i, j}|\nabla \phi|
$$

on the regular grid where is the level-set function. As the function evolves continuously, so does the implicitly represented curve.

The level set function in the foreground region is assigned a positive value while the background region is 
assigned a negative value. Then the $\mathrm{L}_{2}$ difference values of the intensity values inside the local windows which are set at the boundary of the object are computed to decide the speed function $F$.

The speed function $F$ is computed by the following three steps:

1. Set $V_{i, j}^{i n}$ to the minimum value of $\left\|I_{i, j}^{1}-I_{i+l_{1}, j+l_{2}}^{0}\right\|^{2}$ over all integer pairs $\left(l_{1}, l_{2}\right)$ in the window if $\left(i+l_{1}, j+l_{2}\right) \in \operatorname{Object}(\phi=1)$ in the previous frame.

2. Set $V_{i, j}^{\text {out }}$ to the minimum value of $\left\|I_{i, j}^{1}-I_{i+l_{1}, j+l_{2}}^{0}\right\|^{2}$ over all integer pairs $\left(l_{1}, l_{2}\right)$ in the window if $\left(i+l_{1}, j+l_{2}\right) \in \operatorname{Background}(\phi=-1)$ in the previous frame.

\section{3. $F_{i, j}=V_{i, j}^{i n}-V_{i, j}^{\text {out }}$.}

Here, the superscript 1 in $I_{i, j}^{1}$ denotes the current frame, while the superscript 0 in $I_{i+l_{1}, j+l_{2}}^{0}$ denotes the previous frame.

Figure 1 shows how the active contour is moved by the above three steps. The left upper image in Fig. 1 shows the previous frame, while the right upper image shows the current frame. The lower row shows enlarged local regions of the upper row frames.

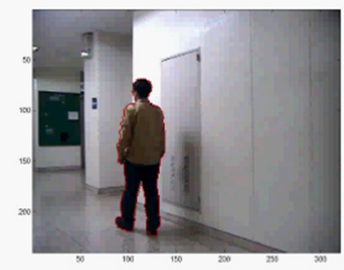

Previous frame

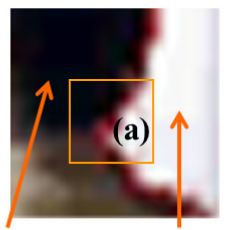

$\phi>0 \quad \phi<0$

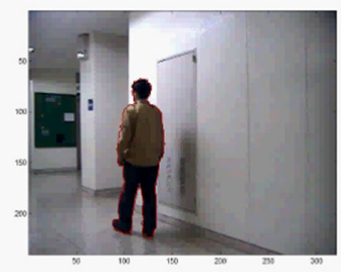

Current frame

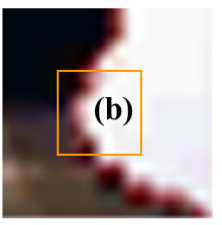

Fig. 3. Showing how the Mansouri model acts: Left : previous frame Right: current frame, (a) background color in the previous frame, (b) background color in the current frame.

It can be seen that the sign of the speed function $F$ is positive if $V_{i, j}^{\text {in }}>V_{i, j}^{\text {out }}$ and negative if $V_{i, j}^{i n}<V_{i, j}^{\text {out }}$. This decides the direction of the motion of the contour, since the sign of $F$ decides whether the value of the level set function at $(i, j)$ decreases or increases as can be seen from (10).

The color at point (b) in Fig. 3 is closer to the background color in the previous frame, i.e., is close to the color at point (a). Therefore, at this point, it becomes that $V_{i, j}^{i n}>V_{i, j}^{\text {out }}$, and the sign of $F$ at this point becomes positive. This makes the value of $\phi$ to decrease at this point, and when $\phi$ becomes negative, then this point becomes a member of the background region. Therefore, the point (b) in Fig. 3 has changed from the foreground region to the background region.

However, a classification can happen if a new color appears in the background region in the current frame which is closer to the foreground region in the previous frame.

Figure 4 shows such a case. Here, compared with colors inside the window (shown as a blue box in Fig. 4 ) in the previous frame, a new color has appeared inside the window in the current frame (indicated by the letter (c)). This color had been occluded by the tracking object, and therefore had not been observed in the previous frame. The problem is that the new color is more close to the object color than the background color in the $\mathrm{L}_{2}$ difference norm sense. Therefore, the Mansouri algorithm regards this color as the object color rather than the background color, and the speed function of the active contour is computed such that it includes this color. As a result the region with this color is included in the object region as can be seen in the right image of Fig. 4.
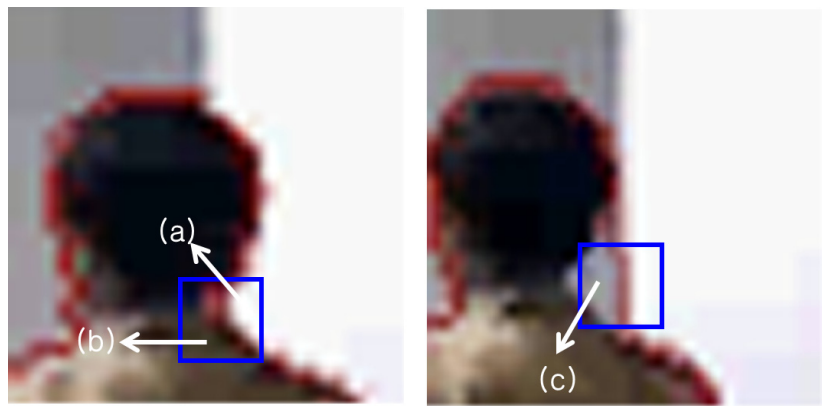

Fig. 4. Showing the case when the Mansouri model acts abnormal Left : previous frame Right: current frame, (a),(b) background and foreground colors in the previous frame, (c) new background color in the current frame.

Once the false region is included in the object region, it remains as the object region, and therefore, the error of the active contour region and the object region increases with time and the tracking fails as can be seen in Fig. 4.

Therefore, a new speed function has to be proposed which can deal with this problem. 


\section{PROPOSED METHOD}

We propose a new speed function which can deal with the above mentioned problem. We let the proposed speed function be

$$
\begin{aligned}
& F(r, t)=\min \left\{\varphi_{t}, 0\right\} \\
& +\max \left\{\varphi_{t}, 0\right\} \int_{B_{a}\left(r_{0}\right)}^{\frac{\sigma}{\varepsilon+\left|I\left(r_{0}, t+\Delta t\right)-I(r, t)\right|}}\left[2 \chi_{R_{t}}(r)-1\right] d r
\end{aligned}
$$

where $B_{a}\left(r_{0}\right)$ denotes a small ball region with radius $a$ around $r_{0}$, and $\chi_{R_{t}}(r)$ is the characteristic function of the region $\left\{r \mid \varphi_{t}(r)>0\right\}$, and $\sigma$ and $\varepsilon$ are small positive values.

To see how the proposed speed function works, we first observe how the term after the $\max \{\}$ function in (3) works. We let this term be

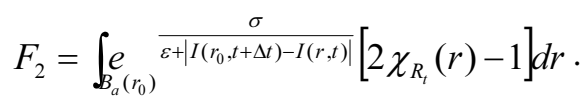

We see that $F_{2}$ is positive if $\left\lfloor 2 \chi_{R_{t}}(r)-1\right\rfloor$ is positive, which is again positive if the point $r$ has been the object region in the previous frame, i.e., $\phi$ has been positive in the previous frame. Meanwhile $F_{2}$ is negative if $\left\lfloor 2 \chi_{R_{t}}(r)-1\right\rfloor$ is negative, which is negative if the point $r$ has been the background region in the previous frame.

The value $F_{2}$ is computed inside the ball region $B_{a}\left(r_{0}\right)$, and has a meaningful value only if $e^{\frac{\sigma}{\varepsilon+\left|\left(r_{0}, t+\Delta t\right)-I(r, t)\right|}}$ is large enough, i.e., if the color value at $r$ in the previous frame and the color value at $r_{0}$ in the current frame are not too different.

The max function multiplied to $F_{2}$ has to be positive if the function $F_{2}$ is to have an effect on the speed function $F(r, t)$. That is, if $\varphi_{t}$ is positive then we let $F_{2}$ to decide whether $F(r, t)$ is positive or negative. If $\varphi_{t}$ is negative then $F(r, t)$ is negative, regardless of the sign of $F_{2}$.

Here, the function $\varphi_{t}$ is the result of bimodal segmentation [8], which we have proposed to compute the Chan-Vese model in a speedy and stable way. The function $\varphi_{t}$ is computed as the minimizer of the following functional:

$$
\begin{aligned}
& E\left(\varphi_{t}\right)=\int\left|\hat{I}(r, t)-a v e_{\left\{\varphi_{t} \geq 0\right\}}\right|^{2} \varphi_{t} H\left(\delta+\varphi_{t}\right) d r \\
& -\int\left|\hat{I}(r, t)-\operatorname{ave}_{\left\{\varphi_{t}<0\right\}}\right|^{2} \varphi_{t} H\left(\delta-\varphi_{t}\right) d r
\end{aligned}
$$

Here, $H$ is the Heaviside function, and $\delta$ is a small positive value. The minimization above leads to a segmentation of $\Omega=\Omega_{\text {in }} \cup \Omega_{\text {out }}=\left\{\varphi_{t}>0\right\} \cup\left\{\varphi_{t}<0\right\}$. Here, we try to force the speed function $F$ to be $F<0$ in the region $\Omega_{\text {out }}$. For this aim, we defined the new difference image $\hat{I}(r, t)$ as

$$
\hat{I}(r, t):=\beta_{1} \exp \left[\sum_{j=1}^{3} \frac{-\beta_{2}}{\left|I(r, t)-\lambda_{j}(t)\right|^{q}}\right]
$$

where $\beta_{1}$ and $\beta_{2}$ are scaling parameters, and $I(r, t)$ is the original image, the incoming frame. In the new image $\hat{I}(r, t)$, the colors corresponding to $\lambda_{j}(t)$ diminish, since the denomitor becomes small. We choose a dominant color $\lambda_{1}(t)$ in such a way that

$$
\lambda_{1}(t)=\arg \sup _{\mu} \int_{\mu-\sigma}^{\mu+\sigma} \phi_{I}(s, t) d s
$$

and

$$
\int_{\lambda_{1}-\sigma}^{\lambda_{1}+\sigma} \phi_{I}(s, t) d s>\alpha|\Omega|,
$$

where $\phi_{I}(s, t)$ denotes the histogram of $I$ at the level $s$ and at time $t$. The supremum of (15) corresponds to the dominant color, while the condition in (16) corresponds to the color that covers a region of reasonable size.

Here, we carefully choose $\delta$ and $\sigma$ that represent the variance and the portion of the image, respectively. We usually choose $\frac{1}{9}<\delta<\frac{1}{2}$.

Similarly, we can choose the second dominant color (if it exists) in such a way that

$$
\lambda_{2}(t)=\arg \sup _{\left|\mu-\mu_{1}\right|} \int_{\mu-\sigma}^{\mu+\sigma} \phi_{I}(s, t) d s
$$

and

$$
\int_{\gamma_{2}-\sigma}^{\lambda_{2}+\sigma} \phi_{I}(s, t) d s>\alpha|\Omega| .
$$

This makes the colors in the new image to diminish, if they are close to $\lambda_{2}(t)$. Similarly, we choose the third dominant color if it exists, and continue in the same way with the fourth color, etc. Using this new image we obtain a function $\varphi_{t}$ as a minimizer of the energy functional in (13). This minimizer is then used to compute the proposed speed function using equation (11). Due to the bimodal segmentation, the major background colors are eliminated from the background region. The minor background colors which are close to the target colors become now segmented to the background, and thus the active contour will not fall into a local minimum. This makes the tracking more stable than the Mansouri model. 


\section{EXPERIMENTAL RESULTS}

Experiments have been performed on a whole image sequence to compare the tracking results of the Mansouri model and the proposed model. Figure 5 shows the tracking results with the Mansouri model. It can be observed that the contour becomes trapped at the region where the background has a new color. As a result, the contour eludes from the true object boundary, and the whole tracking fails.

Figure 6 shows the tracking result with the proposed model. As can be observed, the new speed function can handle the new colors in the background, and as a result, the contour sticks to the object boundary. The tracking becomes more stable than the Mansouri model, as the center of the object region is more accurately computed, and a more accurate boundary of the object is obtained.
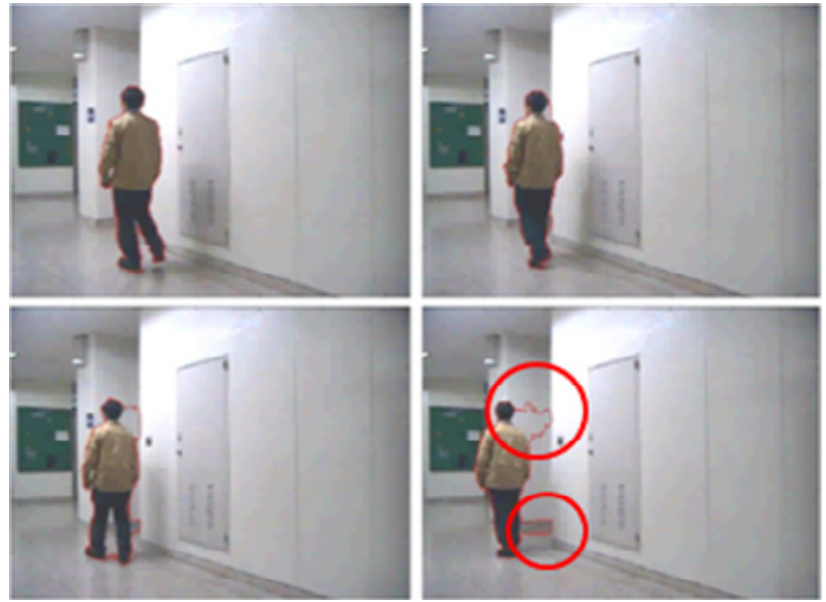

Fig. 5. Tracking result with the conventional Mansouri model. The contour is trapped by the background colors as can be seen in the red circles in the bottom row.
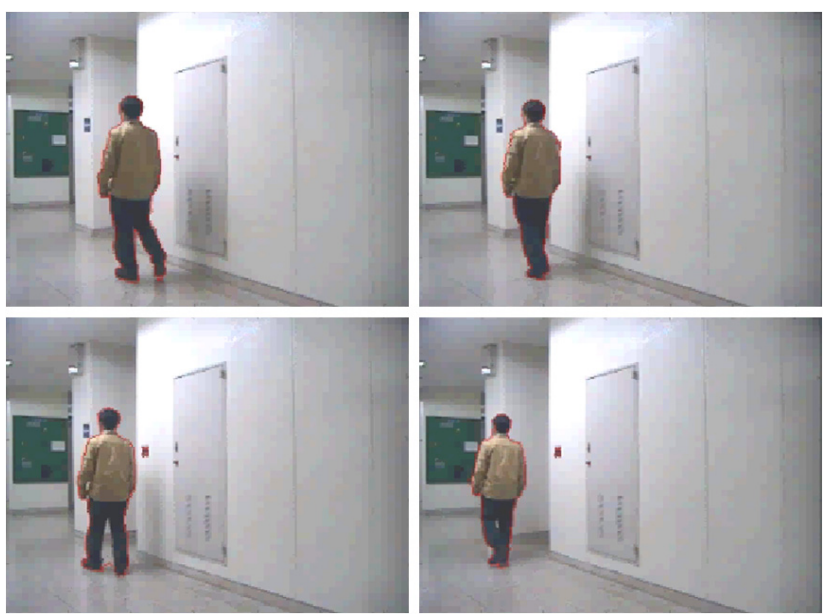

Fig. 6. Tracking result with the proposed model. The contour is not trapped by the background colors as can be seen in the bottom row.

\section{CONCLUSION}

In this paper, we corrected the speed term used in the conventional Mansouri model such that the active contour becomes not trapped by background colors similar to those in the target region. The correction is done by using the bimodal level set segmentation algorithm to determine the dominant background colors. Experimental results verify the performance of the proposed method.

\section{ACKNOWLEDGMENT}

This work was supported by the National Research Foundation of Korea(NRF) grant funded by the Korea government(MEST) (No. 2011-0005614).

\section{REFERENCES}

[1] S. Osher and J. Sethian, "Fronts propagation with curvaturedependent speed: Algorithms based on Hamilton-Jacobi formulations," J. Comput. Phys., vol. 79, pp. 12-49, 1988.

[2] N. Paragios and R. Deriche, "Geodesic active contours and level sets for the detection and tracking of moving objects," IEEE Trans. Pattern Anal. Mach. Intell., vol. 22, no. 3, pp. 266-280, Mar. 2000.

[3] T. Chan and L. Vese, "Active contours without edges," IEEE Trans. Image Process., vol. 10, no. 2, pp. 266-277, Feb. 2001.

[4] Alper Yilmaz, Xin Li, and Mubarak Shah, "Contour-Based Object Tracking with Occlusion Handling in Video Acquired Using Mobile Cameras", IEEE Trans. PAMI, vol. 26, no. 11, pp. 15311536, Nov., 2004.

[5] G. Sundaramoorthi, A. Yezzi, A.C. Mennucci, "Coarse-to-Fine Segmentation and Tracking Using Sobolev Active Contours," IEEE Trans. PAMI, vol. 30, no. 5, pp. 851-864, May, 2008.

[6] Wei Yu, F. Franchetti, Yao-Jen Chang, Tsuhan Chen, "Fast and robust active contours for image segmentation", Proceedings of IEEE ICIP, pp. 641-644, Sep. 2010.

[7] A. Mansouri, "Region Tracking via Level Set PDEs without Motion Computation," IEEE Trans. PAMI, vol. 24, no. 7, pp. 947961, July, 2002

[8] Suk-Ho Lee and Jin Keun Seo, "Level set-based bimodal segmentation with stationary global minimum", IEEE Trans. Image Process., vol. 15, no. 9, pp. 2843 - 2852, Sep. 2006.

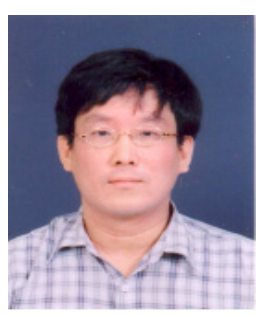

Suk-Ho Lee received his BS, MS, and PhD in electronics engineering from Yonsei University, Korea, in 1993, 1998, and 2003, respectively. He worked as a researcher in the Impedance Imaging Research Center from 2003 to 2006 and was an assistant professor at Yonsei University from 2006 to 2008 . He has been in the Department of Multimedia Engineering at Dongseo University, Busan, Korea, since 2008, where he is now a professor. His current research interests include image and video filtering based on PDEs, medical imaging, and computer vision. 